Pedagogía y Saberes No. 47

Universidad Pedagógica Nacional

Facultad de Educación. 2017, pp. 65-77

\title{
Experiencia y problemas. Educación ciudadana y enseñanza de la filosofía*
}

\section{Artículo de reflexión}

\section{Experience and Problems. Education for Citizenship and Philosophy Teaching}

Experiência e problemas. A educação cidadã e ensino da filosofia

\section{Germán Vargas Guillén ** \\ Raúl Ernesto Meléndez Acuña*** Wilson Ricardo Herrera Romero ${ }^{* * * *}$}

Para citar este artículo:

Vargas, G., Meléndez, R. y Herrera, W. (2017). Experiencia y problemas. Educación ciudadana y enseñanza de la filosofía. Pedagogía y Saberes, (47), 65-77.

* Este artículo reelabora las bases conceptuales que prepararon los autores para la prueba de selección de docentes en el concurso del Ministerio de Educación Nacional, a través de consultoría con el ICFES (2016). Se omiten todos los aspectos técnicos (criterios y preguntas), presentados en la consultoría referida. Se publica con la autorización del ICFES.

** Profesor titular de la Facultad de Humanidades de la Universidad Pedagógica Nacional. Doctor en Educación de la misma universidad. Investigador del grupo Filosofía y enseñanza de la filosofía de la Universidad Pedagógica Nacional.

Correo electrónico: gevargas@pedagogica.edu.co

Perfil ORCID: http:// orcid.org/0000-0001-6156-799X

*** Profesor asociado de la Universidad Nacional de Colombia. Magister y doctor en filosofía de la misma universidad. Investigador del grupo Lógica, epistemología y filosofía de la ciencia de la universidad Nacional.

Correo: remelendezac@unal.edu.co

Perfil ORCID: http:// orcid.org/0000-0003-4158-5829

****Profesor asociado de la Universidad del Rosario. Doctor en filosofía de la Binghamton University. Investigador del Grupo de ética aplicada, trabajo y responsabilidad social, Escuela de Ciencias Humanas, Universidad del Rosario. Correo: wherrer29@gmail.com Perfil ORCID: http:// orcid.org/0000-0002-2363-6998 


\title{
Resumen
}

Este artículo de reflexión parte de las preguntas cómo enseñar filosofía y para qué enseñar filosofía. En él se problematizan los alcances de la enseñanza de la filosofía en torno a los fines de la educación, específicamente, al de la democracia. Se sostiene la tesis de que una educación filosófica es base de la educación ciudadana toda vez que pone a los sujetos, a través de su formación, en condiciones de reflexionar sobre sí mismos, sobre su entorno, y sobre su relación con los otros. El artículo se organiza en tres partes: la primera presenta los fines de la educación como fuente de sentido de la enseñanza de la filosofía en función de una educación filosófica; la segunda, analiza el alcance del concepto de experiencia como eje articulador de los polos (problemas y pensamiento crítico) que tensan la educación filosófica y, la tercera que presenta las conclusiones, señala cómo esta tensión (problemas y pensamiento crítico) puede ser operada en el aula de educación básica.

\section{Palabras clave}

formación; democracia; fines de la educación; pensamiento crítico; ambientes de aprendizaje

\begin{abstract}
This reflection article starts from the questions on how and why philosophy should be taught. It problematizes the scopes of teaching philosophy with regards to the scopes of education and, specifically, of democracy. The thesis is that a philosophical education is the foundation of a citizen education, insofar as it puts the subjects in a position to reflect about themselves, their environment, and their relationship with others. The paper is divided in three parts: the first one presents the goals of education as a source of meaning in the teaching of philosophy in terms of a philosophical education; the second part analyzes the scope of the concept of experience as the articulating axis of the two poles (problems and critical thinking) that strain philosophical education; and the third part that shows a conclusions, present how this tension (problems and critical thinking) can be implemented inside the classroom of basic education.
\end{abstract}

\section{Keywords}

training; democracy; purposes of education; critical thinking; learning environments

\section{Resumo}

Este artigo de reflexão baseia-se nas perguntas: como ensinar filosofia e para que ensinar filosofia. Nele, são problematizadas as dimensões do ensino da filosofia em relação aos fins da educação, especificamente, à democracia. Afirma-se que uma educação filosófica é base da educação cidadã toda vez que põe os sujeitos, através da sua formação, em condições de refletir sobre sim mesmos, sobre o seu entorno, e sobre sua relação com os outros. 0 artigo está organizado em três seções: a primeira apresenta os fins da educação filosófica; a segunda, analisa a dimensão da concepção de experiência como eixo articulados dos polos (problemas e pensamento crítico) que põem em tensão a educação filosófica; e a terceira que apresenta as conclusões, mostra como essa tensão (problemas e pensamento crítico) pode ser operada na sala de aula da educação básica.

\section{Palavras chave}

formação; democracia; fins da educação; pensamento crítico; ambientes de aprendizagem 
i Cómo enseñar filosofía?, es una pregunta que desafía a quienes se encuentran en la tarea de contribuir a los procesos de formación de las nuevas generaciones. Pero la importancia de esta pregunta es subsidiaria de una, acaso precedente, ¿para qué enseñar filosofía? En último término, responder a esta cuestión tiene que ver con los fines de la educación. Puede resultar repetitivo decir que éstos se orientan a la democracia, pero se tiene que insistir en decirlo una vez más. Y, sin embargo, la novedad de la respuesta puede estar asociada al hecho de que una educación filosófica es base de la educación ciudadana toda vez que pone a los sujetos, a través de su formación, en condiciones de reflexionar sobre sí mismos, sobre su entorno, sobre su relación con los otros. Esta educación filosófica es análoga al mínimo que requiere un ciudadano, en materia de formación, en otros campos del saber para poder vivir en el mundo cotidiano: pensamiento matemático, pensamiento físico, pensamiento histórico, pensamiento geográfico, etc. Sin ser geógrafos, todos y cada uno de los ciudadanos necesitan poderse orientar en el espacio, resolver problemas de razonamiento espacial; sin ser matemáticos, todos los ciudadanos tienen que resolver problemas desde muy elementales hasta muy complejos de cálculo aritmético, de razonamientos geométricos, etc.; sin ser historiadores, todos los ciudadanos necesitan saber de su origen, del sentido destinal de su vida; sin ser físicos o químicos, en la vida diaria los sujetos tienen que enfrentar y resolver problemas de esta índole. Todo ello exige una formación básica. Justamente en la formación básica se inserta la pregunta por los fines de la educación filosófica, sobre la contribución de ésta a la vida cotidiana de los sujetos.

Ahora bien, ¿cómo hacer de la formación filosófica una experiencia significativa? Y, entonces, ¿cómo entender, en este contexto, el concepto mismo experiencia? Actividades llevamos a cabo diariamente, pero ¿cuándo adquieren sentido? ¿Cómo se da el paso de un haz de situaciones en las que nos vemos inmersos a la experiencia? Y, más concretamente, ¿cómo se puede fundar la enseñanza de la filosofía en la experiencia? La tesis que se sostiene es que una educación filosófica que no parta de y no retorne a la experiencia garantiza la pérdida del sentido de esta formación para todos los implicados: alumnos, padres de familia, profesores, empresarios, Estado, en fin, actores sociales. Antes bien, partir de y retornar a la experiencia hace de la educación filosófica un valor, un sentido y un proyecto para todos y cada uno de estos.

Al cabo, se puede afirmar que la educación filosófica tiene un anclaje en problemas; y que éstos son fuente para la formación del pensamiento crítico. La enseñanza de la filosofía se articula en estos dos polos: problemas como punto de partida y pensamiento crítico como punto de llegada. Así se tensa el proceso dentro del cual tiene un juego y un rol significativo la historia de la filosofía y el presente viviente; los textos filosóficos, la tradición, y la innovación en el pensamiento. En esta tensión tiene lugar o espacio el rol creativo tanto de aprendices como de enseñantes. En esta tensión se opera, una y otra vez, el papel de cada uno de los actores sociales como protagonistas de su propia comprensión de mundo (de sentido de sí, del entorno, de la relación con los otros).

Los tres pasos que se dan en el artículo, en consecuencia, presentan los fines de la educación como fuente de sentido de la enseñanza de la filosofía en función de una educación filosófica; el alcance del concepto de experiencia como eje articulador de los polos (problemas y pensamiento crítico) que tensan la educación filosófica. Y se concluye mostrando cómo esta tensión (problemas y pensamiento crítico) puede ser operada en el aula en el proceso de la educación básica.

\section{Fines de la enseñanza de la filosofía en Colombia}

La formación ciudadana es el medio por excelencia en el que una sociedad se proyecta hacia el futuro. La cuestión en torno a qué se quiere ser como sociedad y cómo se llega a ello no se puede responder sin tener en cuenta lo que pasa con los procesos educativos en todas las esferas de la sociedad. En este sentido, el qué y para qué de la educación es un asunto que concierne a todos y no solo a quienes están encargados de diseñar las políticas educativas o a los expertos que los asesoran. Ciertamente, son los miembros de la comunidad educativa los que están llamados a establecer y evaluar en cada momento cuáles son los fines de la educación. Empero, esta comunidad no se reduce a los directivos, docentes y estudiantes, sino que alude a todos los actores sociales. Es menester tener en cuenta que la formación ciudadana no se da sólo en la escuela y en la familia, sino también en otros espacios como la empresa, la justa deportiva, los medios de comunicación, los espacios públicos, etc. Por ello, se puede decir que una sociedad democrática, en principio, se concibe como aquella en que los asuntos son decididos por todos los ciudadanos; por ejemplo, el establecimiento de los fines de la formación ciudadana, que definen lo que se quiere ser, deben ser resultado de una deliberación democrática en la que los distintos actores sociales participan y exponen sus posturas y experiencias al escrutinio público.

En el caso colombiano, resulta notorio que en los dos últimos años la educación se ha convertido en una de las preocupaciones centrales de la opinión pública. 
En especial, dos problemas han salido a la palestra de los medios: la calidad de la educación y su papel en la construcción de paz. Si bien estos dos problemas están íntimamente relacionados, hay la tendencia en los medios, y entre ciertos expertos, a reducir la calidad de la educación al cumplimiento de ciertos estándares que se ven reflejados en el desempeño de los estudiantes en las Pruebas Pisa y Saber 11; sin considerar la cuestión de cómo se relaciona la educación con la construcción de paz. A este respecto, no es una exageración afirmar que la persistencia de la violencia en las distintas esferas de la realidad social colombiana indica que algo pasa con la calidad de la formación ciudadana y que ésto es mucho más diciente que lo que muestran las mencionadas pruebas.

Como es bien conocido, en la Constitución de 1991, que ha sido vista por muchos como una especie de pacto de paz, se concibe a Colombia como una sociedad pluralista y democrática. En principio, la Carta expresa un ideal compartido por los colombianos: que es valioso que existan diversos grupos y comunidades con visiones diferentes sobre lo que somos y queremos ser. Ahora bien, al pluralismo le es inherente la diferencia y el desacuerdo. Lo que la Carta establece es que, a través de procesos democráticos, los colombianos se deben poner de acuerdo en torno a cómo resolver aquellos asuntos que requieren de la cooperación de todos, y que contribuyen a la construcción de una sociedad justa en la que podamos llevar a cabo nuestros propios planes de vida, y al mismo tiempo que se respeten los derechos de todos sin discriminación de ningún tipo. En otras palabras, la Constitución parece promover la idea de que la riqueza de la nación está en construir un "nosotros" a partir de las diferencias. Este ideal no fue el resultado de los sueños de unos visionarios provenientes de otros lares, sino que fue fruto de una discusión entre los miembros de la Asamblea Nacional Constituyente que representaban las distintas voces de la sociedad. En estas discusiones, se recogió el clamor de muchos sectores sociales de no suprimir, a través de la violencia, la diferencia; y se planteó la necesidad de tramitar nuestras diferencias a través de vías democráticas.

Una revisión al Informe Final de la Comisión de Memoria Histórica Basta Ya (2013), comprueba de una manera abrumadora que este ideal de la Constitución de 1991 está aún muy lejos de ser parte constitutiva de nuestras formas de interactuar los unos con los otros. Como bien lo señala Marta Nubia Abello, en la introducción al informe, en Colombia "hay un déficit de ciudadanía y de democracia" (p. 23) que se expresa en "un uso arbitrario y maniqueo de los mecanismos de participación y decisión pues se reclama la democracia cuando favorece posturas e intereses propios y se atenta contra ella cuando reconoce los intereses y derechos de los otros" (p. 23). Las causas de este déficit son tanto objetivas como subjetivas. De una parte, tienen que ver con unas estructuras sociales y económicas inequitativas y un sistema político que ha tendido a ser cerrado y excluyente. De otra parte, este déficit de ciudadanía se ha dado en el marco de un sistema educativo que, en lugar de contribuir a integrar a la sociedad y a formar una cultura política más democrática, ha ahondado las diferencias sociales y ha alimentado los prejuicios que justifican la exclusión y la intolerancia.

En este contexto, cabe preguntarse cuál puede ser el papel de la filosofía. Este problema resulta más apremiante si se tiene en cuenta lo que sucede en la actualidad con las Pruebas Saber 11, donde el componente de filosofía ha quedado incorporado a una prueba de Lectura crítica. Esta reestructuración de la Prueba Saber 11 conduce, según el criterio de muchos, a cierta invisibilización de la filosofía y, con ello, se pone en riesgo su ejercicio en el país. Sin embargo, en esta preocupación hay un problema que es mucho más apremiante para quienes se dedican a enseñar filosofía: la calidad y la pertinencia de su enseñanza. En este sentido, cabe preguntarse si aprender algo de filosofía contribuye con la educación y en qué medida lo hace. A este respecto, el hecho de que existan sistemas educativos que contemplan la enseñanza de la filosofía a nivel básico (como los casos de Brasil y Colombia) y sistemas que no la contemplan (como Estados Unidos, Canadá y España) indica, en principio, que no hay una respuesta unánime sobre la pertinencia de la enseñanza de la filosofía en la Educación Media. Ahora bien, se podría decir que la respuesta a esta pregunta no puede hacerse de manera abstracta y general, sino que depende de los fines a los que apunta la educación en cada sociedad. En otras palabras, podría decirse que el papel que cumpla la enseñanza de la filosofía está ligado a cómo se conciba, en cada sociedad, el proceso educativo en estos niveles. Así, siguiendo a Amy Gutmann (2001), dado que la educación es un medio a través del cual se forman los ciudadanos del futuro, la cuestión sobre sus fines se enmarca dentro de una pregunta más general sobre qué sociedad se quiere en el futuro. Como ya se dijo, en el contexto de una sociedad democrática, un asunto como éste es una decisión política que debe ser tomada por los ciudadanos. Consecuentemente, sabiendo que las generaciones presentes deciden el destino de las generaciones futuras, es fundamental que se tenga claridad sobre lo que está en juego y, por ende, es fundamental realizar una reflexión filosófica sobre lo que significa la educación y sobre los fines que 
ella persigue, puesto que ésta puede dar luces a los ciudadanos para que sus decisiones sean razonables.

En Colombia, tanto en la Constitución de 1991 como en la Ley General de Educación (Ley 115 de 1994), se establece una diversidad de fines para el sistema educativo. Entre ellos, hay tres que son claves para entender el rol que puede tener la enseñanza de la filosofía en la formación ciudadana. En primer lugar, la Ley establece que la educación en Colombia tiene como eje la formación de una ciudadanía respetuosa de los derechos fundamentales. En este sentido, se afirma que la educación tiene como fin "la formación en el respeto a la vida y a los demás derechos humanos, a la paz, a los principios democráticos, de convivencia, pluralismo, justicia, solidaridad y equidad, así como en el ejercicio de la tolerancia y de la libertad" (art. 1, numeral 2). En segundo lugar, en la misma normativa se señala que a través del proceso educativo se busca formar personas que puedan ser competentes o productivas, en otras palabras, formar lo que los economistas llaman un capital humano calificado. Al respecto, la Ley establece que la educación debe promover "en la persona y en la sociedad la capacidad para crear, investigar, adoptar la tecnología que se requiere en los procesos de desarrollo del país y le permita al educando ingresar al sector productivo" (art. 5, numeral 13). En tercer lugar, también se concibe la educación como un espacio para que se dé "el pleno desarrollo de la personalidad sin más limitaciones que las que le imponen los derechos de los demás y el orden jurídico, dentro de un proceso de formación integral, física, psíquica, intelectual, moral, espiritual, social, afectiva, ética, cívica y demás valores humanos" (art. 5, numeral 1). En términos generales, se puede afirmar que estos tres propósitos corresponden a lo que en una sociedad democrática de corte liberal republicano se espera de la ciudadanía, a saber: que tenga autonomía personal y moral, que sea respetuosa de los derechos de los otros, que sea solidaria y que sea productiva.

Hechas las anteriores consideraciones, la cuestión sobre la enseñanza de la filosofía no se puede abordar solamente desde la pregunta por el valor intrínseco del quehacer filosófico, sino que debe considerar el para qué de la filosofía en relación con una sociedad como la nuestra. Hay que preguntarse cómo se vincula la enseñanza de la filosofía con la conformación y mantenimiento de una sociedad que, al atenerse a la ley, concibe a sus presentes y futuros ciudadanos como libres, iguales, solidarios y eficientes. En este punto es importante advertir que estos ideales no necesariamente son compatibles entre sí; las exigencias del trabajo en ciertos contextos pueden ir en contravía de un ciudadano autónomo y crítico, la eficiencia y la meritocracia asociada con ella en muchas ocasiones no es compatible con la solidaridad y la equidad.

Quizá la contribución que puede hacer la filosofía se puede inferir de tres tareas que ella, a lo largo de su historia, ha pretendido asumir y al mismo tiempo paradójicamente ha cuestionado. En primer lugar, cualquiera que sea la corriente filosófica, se reconoce que la tarea reflexiva propia de la filosofía se ha dado en diálogo crítico con la tradición filosófica. El aprendizaje filosófico, que nunca culmina en tanto que es siempre incompleto, tiene como exigencia primordial la comprensión de una serie de textos que se han ganado el mote de clásicos. Como lo sugiere Gadamer (1978, p. 331), a pesar del paso del tiempo, lo que se dice en esos libros aún nos interpela y, quizá lo más asombroso, es que, a medida que pasan los años, quienes asumen el reto de seguirlos leyendo, sacan de ellos nuevas interpretaciones que dan luces sobre la complejidad de los problemas contemporáneos y motivan a buscar, desde su lectura, nuevas respuestas. En este marco, en la mayoría de los programas de filosofía, o al menos en los más reconocidos, hay unas prácticas pedagógicas ya centenarias, como la del llamado seminario alemán y el diálogo socrático, que se han probado exitosamente en su esfuerzo por enseñar a los estudiantes a leer los textos filosóficos que, por la naturaleza de las cuestiones que tratan, retan su capacidad reflexiva.

Así, la enseñanza de la filosofía puede ayudar a los educandos, incluyendo a los que no quieren ser filósofos, a que logren poner en práctica, mediante la lectura, la capacidad de tomar en serio su existencia y respetar las diversas aproximaciones que se encuentran en la tradición a diferentes problemas. De esta manera, en la filosofía, la lectura no es simplemente un acto comunicativo fundamental, sino que también se erige como un acto ético que implica el reconocimiento y respeto de la pluralidad de opiniones que se encuentran en diferentes épocas y latitudes.

En segundo lugar, la reflexión filosófica, que a lo largo de su historia ha estado más centrada en cuestionar que en dar respuestas definitivas, tiene una pretensión educativa, a saber, formar seres autónomos y autorreflexivos. Aunque la mayor parte de los seres humanos no practica la filosofía como una profesión, esto es, como una actividad para ganarse la vida, a todos, de una manera u otra les conciernen muchos de los problemas que trata la filosofía. Por ejemplo, el cuestionamiento por la identidad de cada quien (¿quién soy yo?) es un problema que ha sido recurrente en la historia de la filosofía y también es un interrogante que cada uno se hace al menos una vez en su vida (como es el caso de la adolescencia). 
Pero quizá lo más importante, en relación con lo que se ha tratado, es que el esfuerzo central de la filosofía está en analizar los problemas fundamentales de cada presente histórico. Al estar en Colombia, en donde sale a la palestra por doquier la barbarie en que los ciudadanos han vivido en todos estos años, una cuestión urgente para la formación de éstos es ¿cómo hacer frente a los desacuerdos, que como democracia no simplemente se deben aceptar sino celebrar, sin acudir a la violencia o al acallamiento de la deliberación reflexiva?

En La educación después de Auschwitz, Theodor W. Adorno (1998), plantea una tarea para la educación que le es inherente también a la enseñanza de la filosofía y que resulta pertinente para el caso colombiano: la única fuerza verdadera contra el principio de Auschwitz sería la autonomía, si se permite valerse de la expresión kantiana; la fuerza de reflexionar, de autodeterminarse, de no entrar en el juego (p. 83). En este mismo texto, Adorno advierte que a la formación en la autonomía le es inherente una actitud crítica frente a lo que sucede, al statu quo, actitud que está fundada en la sensibilidad y el compromiso con las necesidades y derechos de los otros. Esta referencia a Adorno permite ver claramente que la enseñanza de la filosofía tiene el deber de contribuir en la formación de una ciudadanía autónoma y solidaria.

En tercer lugar, como lo señaló Sócrates, una buena vida es una vida reflexiva. Una tarea fundamental de la educación es que el estudiante pueda encontrar una forma de vivir y de estar en el mundo en la que pueda realizarse. En otras palabras, la educación tiene como propósito generar las condiciones necesarias, aunque no suficientes, para que los estudiantes se realicen o, mejor, para que hagan esa búsqueda por sí mismos sin imposiciones externas. En ese esfuerzo por encontrar una vida propia, el quehacer de la filosofía en la escuela resulta invaluable en tanto que la filosofía ha sido siempre una invitación a no dejarse llevar por la corriente, por lo que dice la autoridad o por las tendencias y opiniones mayoritarias. Los textos filosóficos, cuando son tomados en serio, pueden despertar en el estudiante una actitud crítica hacia sí mismo que le puede ayudar a descubrir, pese al plan de vida que se trace, que la existencia es contingente y que siempre está a merced de los otros.

Así, pues, como un ciudadano tiene que contar con una preparación básica en pensamiento matemático o físico, de igual modo requiere una educación filosófica que le habilite para reflexionar sobre sí mismo, sobre su entorno, sobre su relación con los otros. Al cabo, la educación filosófica no toca solo con la educación ciudadana desde el punto de vista de la relación de cada quien con los otros, sino de la autocomprensión de cada quien con respecto a su propio sentido de vida y a los proyectos que se traza como derroteros de su existencia.

\section{El papel de la experiencia en la enseñanza de la filosofía}

La enseñanza en general, y la enseñanza de la filosofía en particular, requieren apelar tanto a la experiencia como al concepto de experiencia para ser ejecutada en dirección del aprendizaje significativo. El concepto "experiencia" cuenta con una larga tradición en filosofía (Aristóteles, Bacon, Hume, Locke, Kant, Husserl, Dewey, entre otros); sin embargo, si hay algo que pueda objetarse a los profesores que enseñan filosofía es que vuelven la espalda a la experiencia cotidiana. A veces parece que la enseñanza se dirigiera más a las "mentes", al "entendimiento", a la "inteligencia" o a la "razón" que a las personas de "carne y hueso" que están ahí, en el aula, que viven y experimentan el mundo.

En vista de esta necesidad de vincular la enseñanza de la filosofía tanto al concepto de experiencia, como a la experiencia efectiva de los sujetos en su mundo de la vida, se recurre aquí a algunas de las nociones de J. Dewey consignadas en Experiencia y educación (1967).

\section{Experiencia}

La articulación de la experiencia del educando con el saber disciplinar enseñado por el educador es fundamental para que acontezca el aprendizaje. En la enseñanza de la filosofía es indispensable que el profesor logre reconocer, conectar y organizar los problemas que suscitan las diferentes experiencias de los estudiantes con los problemas filosóficos. Dicho de otro modo, si el profesor logra reconocer y organizar los problemas que inducen las experiencias o vivencias de los estudiantes a través del saber filosófico, entonces devienen el aprendizaje y la motivación, en la medida en que el estudiante encuentra en este saber elementos para resolver o lidiar con los problemas surgidos de sus vivencias.

¿Cómo entra en juego la experiencia? Para Dewey: "[...] las experiencias previas han cambiado las condiciones objetivas bajo las cuales tienen lugar las experiencias subsiguientes" (1967, p. 40); esto es, no solo son ingrediente del mundo, sino que abren horizontes o perspectivas de ser. De hecho, se puede decir que la experiencia es entorno de todo lo que se vive como sujeto; pero, igualmente, que la continuidad de una a 
otra experiencia se articula con el sentido, los propósitos o los fines que se persiguen; así, entonces, “[...] vivimos, del nacimiento a la muerte, en un mundo de personas y cosas, que [...] es lo que es por lo que han hecho y transmitido las actividades anteriores" (p. 40).

Un proceso educativo centrado en la experiencia implica interacción, pues la solución de problemas abarca el diálogo con los otros en la medida en que ellos pueden brindar elementos que, a partir de su experiencia, pueden ser valiosos para encontrar una solución al problema en cuestión. Cuando un sujeto quiere solucionar un problema se ve en la necesidad de identificar en los otros (v. gr. en la tradición) el tratamiento que se la ha hecho al mismo problema al que aquel se ve enfrentado actualmente.

En este contexto, en la enseñanza en general y en particular en la enseñanza de la filosofía, la experiencia es fundamento, en ella se acude a las vivencias que tienen, uno a uno, los individuos. Mediante la experiencia se estructura y direcciona lo vivido en función de propósitos y de fines, para los cuales los sujetos diseñan proyectos. Éstos no solo implican a los individuos, sino que se articulan con los demás, con la comunidad. Así, la enseñanza de la filosofía comienza por garantizar que la experiencia se trabaje como un elemento integrante del componente pedagógico-didáctico. De lo que se trata en esta enseñanza es de especificar cómo el profesor puede hacer la conexión entre la filosofía y las experiencias de los estudiantes; en este marco, el profesor pone en juego la capacidad para formular preguntas que organicen las experiencias de sus estudiantes para lograr que ellos se percaten de un problema que pueden solucionar a través de la filosofía y del filosofar.

Consecuencia de introducir la experiencia en el proceso educativo es que la relación entre el profesor y el estudiante se modifica. Aunque el papel de la tradición es fundamental en el proceso educativo, cambia la manera como se ejecuta su rol. No se trata de que el profesor se exhiba como un sabio que domina la tradición sin importar lo que pase con las experiencias de los estudiantes; lo que interesa es que el estudiante pueda usar la tradición para solucionar los problemas hallados en sus experiencias. Por esta razón, el profesor es visto como un creador o diseñador de ambientes de aprendizaje que permitan conectar la experiencia del estudiante con el saber cribado a lo largo de la tradición.

En el caso de la enseñanza de la filosofía, el profesor motiva la participación de los estudiantes al conectar los problemas que surgen de sus experiencias con la tradición filosófica. Una manera de lograr esta conexión se realiza mediante el preguntar, pues si el profesor plantea cuestiones adecuadas al estudiante frente a una determinada situación, entonces conduce a éste a reestructurar su experiencia en pro de esa pregunta y, a su vez, induce al mismo a que evidencie que el problema que implica la cuestión formulada se relaciona o se vive en su situación actual; así el estudiante llega a ver elementos en la tradición filosófica que contribuyen a pensar soluciones a su situación. Sin embargo, no toda pregunta del estudiante visibiliza un problema, por lo tanto, es preciso evaluar la capacidad que tiene el profesor de replantear o reformular las preguntas -con base en su experiencia y su preparación académica-de forma que logre que el estudiante vea en su experiencia un problema y que el filosofar le sirva para encontrar posibles soluciones a ésta. En este sentido, "la experiencia [...] influye en la formación de actitudes [,] de deseo ${ }^{1}$ y de propósito" (Dewey, 1967, p. 40).

\section{Principios}

En este marco de referencia se rechaza la educación tradicionalista, pues en ella el estudiante es tomado como un "ser" pasivo, que debe acumular y memorizar diversos datos que no están conectados, efectivamente, con sus vivencias y experiencias; el profesor es visto como una figura de autoridad incuestionable que se encarga de transmitir los datos que han sido considerados valiosos por la tradición.

Si se mantiene esta concepción del proceso educativo, difícilmente la experiencia cumple un rol fundamental, pues se cree que el aprendizaje puede acontecer sin tener en cuenta la experiencia del educando. En esa perspectiva, la educación o el proceso educativo se reduce a agregar, transmitir o entregar un cúmulo de saberes que, al estar desconectados de la experiencia, son externos al sujeto y nada tienen que ver con su vida. No obstante, Dewey muestra que para que el proceso educativo direccione la vida de los sujetos, la experiencia debe ser el punto de partida para todo proceso de formación. En último término, si la educación tiene en cuenta la experiencia del sujeto, entonces se convierte en un proceso que le ayuda a vivir porque, por ejemplo, le suministra elementos para resolver sus problemas vitales. Cuando la educación está dirigida a las experiencias de los sujetos permite que éstos "avancen" porque aprenden, entre otras cosas, a solucionar los problemas inmersos en

1 El deseo es un tema que urge reflexión en el contexto de la enseñanza de la filosofía y de la educación filosófica. Una característica de la puesta en juego de esta categoría es que el profesor de filosofía tiene que preocuparse más que por los contenidos por propiciar el deseo de saber en sus estudiantes (cf. Vargas, 2016, pp. 13-39). 
sus situaciones vitales con ayuda de los diferentes saberes que brinda la escuela.

El paso a una educación progresista, basada en la experiencia, parte de reconocer que "los principios [...] para estructurar esa teoría” (Dewey, 1967, p. 31) son los de continuidad e interacción. "Los principios de continuidad e interacción no se pueden separar uno de otro" (p. 47). Ellos dan lugar a la organización de la experiencia.

Primer principio: "continuidad o continuidad experiencial" (Dewey, 1967, p. 31); con él se indica que la educación se orienta a formar el "hábito" (p. 34) o "modo más o menos fijo de hacer las cosas"; a formar "actitudes [...] emocionales e intelectuales" (p. 34). Así, la continuidad es "criterio de diferenciación" (p. 34 ) entre los diversos conjuntos más o menos aislados de actividades o de vivencias y, en rigor, este principio constituye la experiencia. De la continuidad se deriva el "movimiento progresivo" (p. 32). "[...] el principio de continuidad de la experiencia significa que toda experiencia recoge algo de lo que ha pasado antes y modifica en algún modo la cualidad que viene después" (p. 35). La continuidad es "crecimiento", implica "crecer" (p. 35). Esto lleva a concebir "el crecimiento como educación y [...] la educación como crecimiento" (p. 36). Desde esta manera de entender este principio o criterio (continuidad) se puede evaluar la experiencia con la pregunta: “ $¿$ Crea esta forma de crecimiento ulterior o establece condiciones para un crecimiento ulterior o establece condiciones que impide a la persona que ha crecido en esta dirección particular las ocasiones, estímulos y oportunidades para el crecimiento en nuevas direcciones?" (p. 36). Si este crecimiento opera de manera indefinida, de una experiencia a otra, entonces se puede calificar como educativa; si no lo logra, en cambio, se califica de antieducativa. "[...] el principio de continuidad se aplica [...] a todos los casos", pero se aplica de manera diferencial a todos ellos (p. 37).

Segundo principio: "interacción con [...] estados internos inmediatos" (Dewey, 1967, p. 44). Este principio permite "interpretar una experiencia en su función y fuerza educativa” (p. 44). "[...] las condiciones objetivas y las internas [en su interacción] [...] constituyen lo que llamamos una situación" (pp. 44-45). "[...] los individuos viven un mundo [...], viven en una serie de situaciones" (p. 46). Aquí "en" "[s]ignifica que existe una interacción entre un individuo y objetos y otras personas. Las concepciones de situación y de interacción son inseparables unas de otras. [...] experiencia es [...] una transacción entre un individuo y lo que [...] constituye su ambiente, y [...] éste último consiste en personas [...]; juguetes [...]; o [...] libro[s]; o [...] materiales de un experimento [...]" (p. 47). De aquí se deriva la "total estructuración social de las situaciones en que se halla la persona" (p. 49); en último término, esta "estructuración" es la aplicación del principio de interacción.

\section{Organización}

Toda experiencia se debe organizar y si bien en ésta ya hay problemas que no son inventados, estos deben llegar a ser clarificados y estructurados; un cúmulo de acciones o actividades no es, necesariamente, una experiencia; ésta implica organización. Una posible manera de activar la organización es mediante la pregunta; en el caso de la filosofía: mediante la pregunta filosófica. Ésta conduce al estudiante a dialogar con la tradición, pues en ella sus interrogantes vitales ya han tenido tratamiento, han sido materia de la experiencia de otros individuos.

Toda experiencia implica continuidad, puesto que se da en un horizonte temporal de solución: si un agente se enfrenta con una situación nueva para solucionarla apelará a experiencias pasadas -propias o ajenas, en este último caso: en diálogo con la tradición-y, a su vez, se proyectará en una situación futura-como individuo, en relación con los otros-. La experiencia tiene continuidad porque el sujeto puede avanzar en la medida en que puede solucionar situaciones nuevas a partir de la conexión de sus experiencias (apelación al pasado y proyección al futuro).

Una variedad de vivencias (o de actividades, de eventos, de movimientos) por sí mismas no constituyen experiencia; antes bien, se requiere que ésta llegue a alcanzar "organización dentro de la experiencia" (Dewey, 1967, p. 16). “organización progresiva” (p. 17); tanto "el género de organización" (p. 30) como la "conexión orgánica" (p. 92) entre diversas vivencias es lo que toma el carácter de experiencia. Entonces, "[...] toda experiencia debe hacer algo para preparar a una persona para ulteriores experiencias de una calidad más profunda y expansiva. Este es el sentido del crecimiento, la continuidad y la reconstrucción de la experiencia" (p. 52). Con esta última expresión (reconstrucción de la experiencia) se apunta al hecho de que no se trata de tomar ciegamente toda vivencia previa de los individuos, de sacralizar ese haber previo, sino de darle intencionalmente un nuevo sentido a la luz de unos fines, en dirección de la realización de un proyecto -que vale para uno y vale para todos-.

Vista en su organización, la "experiencia es una fuerza en movimiento. Su valor solo puede ser juzgado sobre la base de aquello hacia lo que se mueve" (Dewey, 1967, p. 38) o de los fines que se persigue con ella. "Es misión, pues, del educador ver en qué 
dirección marcha la experiencia" (p. 39). Pero, inherente a la organización que torna las vivencias en experiencia es que toma la vida comunitaria y social como su entorno: la "propia experiencia" y "toda experiencia es últimamente social: [...] representa contacto y comunicación" (p. 39).

Y, no obstante, punto de partida para la organización de la experiencia es que reconoce a los individuos en sus potencialidades, en su singularidad: "Al maestro [...] incumbe [...] conocer las potencialidades para dirigir a los alumnos a nuevos campos que se relacionan con sus experiencias ya tenidas, y a usar este conocimiento como criterio para seleccionar y disponer de las condiciones que influyen en su experiencia presente" (Dewey, 1967, p. 96). Organización equivale, pues, a estructuración intencional de las vivencias en dirección de fines que solo se conquistan en la vida en común: "La solución de este problema requiere una filosofía bien meditada de los factores sociales que operan en la constitución de la experiencia individual" (p. 18).

La organización o su ausencia es un criterio para diferenciar lo que es de lo que no es educativo: "Una experiencia es antieducativa cuando tiene por efecto detener o perturbar el desarrollo de ulteriores experiencias. [...] [Cuando restringe] las posibilidades de tener una experiencia más rica" (Dewey, 1967, p. 22). "[...] las experiencias pueden estar desconectadas unas de otras" (p. 23), en este sentido, tendrían más el carácter de actividad que de experiencia; para arribar a esta última se exige organización.

\section{Problemas}

¿Cómo se activa la organización, esto es, la conversión de vivencias (o de actividades, de eventos, de movimientos) en experiencia? ¿Cuál es, pues, el punto de partida de la experiencia, de su organización? En resumidas cuentas, ese punto de partida lo constituyen los problemas: "[...] surgen los problemas si una experiencia dada [...] conduce a un campo que ya no es familiar" (Dewey, 1967, p. 100); en este sentido, los problemas son un tránsito de lo conocido a lo desconocido, de las habitualidades a nuevas formas de vida (pensar, actuar, ser); así, entonces, “[...] los problemas son el estímulo para pensar" (p. 100).

Relativizar las formas de vida es lo que lleva a cabo el reconocimiento de problemas, el problematizar: "[...] las condiciones que se encuentran en la experiencia presente deben utilizarse como fuentes de problemas [, ésta] es una diferencia que diferencia a la educación basada en la experiencia" (Dewey, 1967, p. 100): organizar, dar continuidad e interacción a la experiencia implica recurrir o basarse en problemas; éstos son el punto de partida del diseño de los ambientes de aprendizaje.

Para atender las diferencias individuales hasta convertirlas en proyectos que se realizan en la vida compartida, de un lado; $y$, de otro, atender los problemas comunes o comunitarios que se asumen desde las perspectiva de los diferentes individuos: los problemas aparecen como enlace o interfaz. Así, es "responsabilidad del educador atender [...], primero, que el problema surja de las condiciones de la experiencia que se tiene en el presente y que se halla dentro de la capacidad de los alumnos y, segundo, que sea tal que despierte en el que aprende un deseo activo de información y de producción de nuevas ideas" (Dewey, 1967, pp. 100-101). Así pues, producción exige tanto un arraigo en el mundo social compartido como una puesta en juego de las capacidades, intereses y motivaciones individuales. En fin, en una educación que se basa en presentar una y otra vez "nuevos problemas [, el] proceso constituye una espiral continua" (p. 101), unos problemas conducen a otros y así indefinidamente.

\section{Maestro: diseñador de ambientes de aprendizaje}

Los ambientes de aprendizaje se conforman, al menos, de los siguientes aspectos:

1. El profesor reconoce tanto las condiciones objetivas como los intereses particulares que atraviesan las experiencias de los educandos.

2. Por medio de preguntas lleva al estudiante a que reestructure su experiencia: a) encuentre un problema que puede solucionar, en este caso, con elementos que le brinda la filosofía; b) caracterice una solución; c) planifique su ejecución.

3. Este proceso debe ser progresivo en la medida en que el estudiante en cada situación nueva es capaz de utilizar experiencias pasadas, en las cuales también usó determinados saberes que le permitan remediar la situación actual a la par que se proyecta en una situación futura.

4. Desde luego, el papel del profesor (como parte del ambiente de aprendizaje) radica en identificar la situación del estudiante y saber qué pregunta puede conducir o reconducir la experiencia de éste para que la pueda organizar con la identificación de un problema que vivencia y que debe clarificar y solucionar, en este caso, con ayuda de la filosofía.

La enseñanza, entendida como diseño de ambientes de aprendizaje, comienza por "[...] presentar nuevos problemas, [...] estimular nuevos métodos de observación y de juicio" (Dewey, 1967, p. 95). Desde luego, esta orientación concibe que: "El educador es responsable del conocimiento de los individuos 
y del conocimiento de las materias que lleven a una organización social, una organización en la que todos los individuos tengan una oportunidad de contribuir algo y en que las actividades en que todos participen sean el principal sostén del control" (p. 65). Este diseño no se ejecuta con miras al individuo aislado, sino en su interrelación con los demás; y, no obstante al "crear una experiencia valiosa [...] los educadores" consideran la singularidad de los estudiantes desde el punto de vista de "las capacidades y propósitos" (p. 49) de éstos.

Específicamente "[e]l ambiente [...] es cualquier condición que interactúa con las necesidades, propósitos y capacidades personales para crear la experiencia que se tiene" (p. 47).

En el diseño de ambientes de aprendizaje

[u]na responsabilidad primaria de los educadores consiste en que [...] deben conocer [:]

$[\bullet]$ el principio general de la formación de la experiencia por las condiciones del ambiente, [...]

[•] qué ambientes conducen a experiencias que faciliten el crecimiento. [...]

[•] cómo utilizar ambientes físicos [pupitres, pizarrones, campo escolar, etc.] y sociales [condiciones de la comunidad local, físicas, históricas, económicas, profesionales, etcétera] que existen, a fin de extraer de ellos lo que poseen para contribuir a fortalecer experiencias que sean valiosas. (Dewey, 1967, p. 42).

En fin, diseñar estos ambientes consiste en "estructurar esquemas de educación" (Dewey, 1967, p. 42); y en ellos,

[1]a misión [del educador] es preparar aquel género de experiencias que [incitan la] actividad [del alumno]. [...] toda experiencia continúa viviendo en experiencias ulteriores. [...] el problema central de una educación basada en la experiencia es seleccionar aquel género de experiencias presentes que vivan fructífera y creadoramente en las experiencias subsiguientes. (p. 25).

En la perspectiva del diseño de ambientes de aprendizaje "[...] un educador [...] debe ser capaz de juzgar qué actitudes conducen realmente a un desarrollo continuado y cuáles son perjudiciales" (Dewey, 1967, p. 40) para que los estudiantes logren la reestructuración de la experiencia, en fin, para que desplieguen sus capacidades. Esto exige, de parte del maestro, una comprensión simpática "de los individuos como tales individuos que le dé una idea de lo que ocurre en las mentes de los que están aprendiendo" (p. 40). Así, para el maestro "[d] escubrir lo que realmente es simple y actuar sobre lo descubierto es una tarea" (p. 29) esencial al diseño de los ambientes de aprendizaje.
Del mismo modo, el diseño de ambientes de aprendizaje requiere: "[...] una teoría de la experiencia, [que ofrece] una dirección positiva para la selección y organización de los métodos y materiales educativos apropiados [...]" (Dewey, 1967, p. 29).

Inherente al diseño y la puesta en ejecución de los ambientes de aprendizaje es que "[...] el educador considera a la enseñanza y al aprender como un proceso continuo de reconstrucción de la experiencia" (Dewey, 1967, p. 113); mediante ésta se desacralizan, se resignifican, se resemantizan: las habitualidades, los saberes previos, con que llegan los individuos a la escena educativa.

\section{Control}

Esencial al diseño de ambientes de aprendizaje que parta y retorne al mundo de la vida efectivamente experimentado por quienes lo integren, es el control. Esto implica que "[...] los planes y proyectos educativos que consideran a la educación en la forma de vida-experiencia, están [...] obligados a estructurar y adoptar una teoría inteligente [o] una filosofía de la experiencia" (Dewey, 1967, p. 57) que pone el énfasis en que los estudiantes enriquecen el ambiente de aprendizaje y que del mismo obtienen elementos que les permite reestructurar los sentidos que han dado a sus vidas, a los hechos, a los proyectos posibles. De ahí que se pueda afirmar que "la educación se fundamenta en la vida-experiencia" (p. 57), que no se trata sólo de un ambiente para que se instruya, sino para que se dé, paso a paso, un nuevo sentido a la vida propia y a la vida con los otros. Es en ese arraigo con la vida-experiencia que deviene o sobreviene el control: autorregulación de los individuos, control social o intersubjetivo.

La educación tiene que llevar a difundir y a apropiar "modos de control" (Dewey, 1967, p. 59), como acontece en los juegos. "Los juegos suponen reglas y estas reglas ordenan su conducta. Los juegos no se realizan al azar o por una sucesión de improvisaciones. Sin reglas no hay juego. [...] las reglas son una parte del juego" (p. 59). “[...] las reglas tienen la sanción de la tradición y de los precedentes" (p. 60). Los individuos participan, se integran, tienen experiencia común que lleva al "control de las acciones" (pp. 60-61). El "control" se ejerce en "situaciones en que todos toman parte" (p. 62). Al participar, los individuos ponen en juego su "voluntad" (p. 62). En un contexto tal "el maestro reduce a un mínimo las ocasiones en que ha de ejercer su autoridad de un modo personal" (p. 62).

En el control una y otra vez tiene sentido apelar a la razón, pero con ella más alude a la preferencia, "por qué preferimos" (Dewey, 1967, p. 32), que a la causa. 
Una teoría inteligente, como la que refiere Dewey, requiere que el control devenga de la razón, de la clarificación de motivos y preferencias, que permite que cada quien regule su conducta. De ahí que se pueda aseverar que "la democracia es la mejor de todas las instituciones sociales" (p. 32); la vida democrática en sociedad, políticamente considerada, es un fin de la educación que permite la construcción racional o inteligente del control tanto individual como social.

La motivación, que deviene de la razón, de una teoría inteligente, aparece como expresión del control, en el cual "[1] a actitud más importante que se puede formar es la de desear seguir aprendiendo. [Este es] [...] el precioso don de la capacidad para aprender por la experiencia" (Dewey, 1967, p. 54).

\section{La enseñanza de la filosofía basada en problemas y el desarrollo del pensamiento crítico}

A pesar de que hay diferentes maneras de concebir y practicar la enseñanza de la filosofía, las cuales se apoyan en diferentes perspectivas acerca de esta disciplina, parece haber un acuerdo mayoritario acerca del valor y utilidad de dicha enseñanza para la ejercitación y el desarrollo del pensamiento autónomo y crítico (véase La Filosofía, una escuela de libertad, p. 72). Pero, ¿qué quiere decir, más precisamente, pensar por sí mismo y de modo crítico? Y ¿cómo la enseñanza de la filosofía puede ayudar a fortalecer esta capacidad en los alumnos?

Para tratar de aclararlo, se precisa enfatizar algo que se mencionó anteriormente: que, en general, la actividad que llamamos pensar suele estar motivada por el deseo o el afán de superar una dificultad, de resolver un problema. Como lo subraya John Dewey en Experiencia y educación:

No surgen problemas si una experiencia dada no conduce a un campo que ya no es familiar, y los problemas son el estímulo para pensar. Que las condiciones que se encuentran en la experiencia presente deben utilizarse como fuentes de problemas es una característica que diferencia a la educación basada en la experiencia de la educación tradicional. Pues en ésta los problemas se plantean desde afuera. El crecimiento depende de la presencia de dificultades a vencer en el ejercicio de la inteligencia. (1967, p. 100).

Dewey formula en este pasaje una objeción contra la educación tradicional que puede hacerse, en particular, contra la enseñanza tradicional de la filosofía. En ella, los problemas que se discuten no surgen de la experiencia del alumno, sino que se plantean, por así decirlo, desde fuera y se imponen al estudiante. Impuestos de este modo resulta muy difícil que el alumno no los viva como problemas ajenos, en los cuales no está genuinamente interesado. La discusión de estos problemas despertaría en el alumno un cierto interés por entender cómo otros pensaron para tratar de resolverlos, pero no un estímulo a pensar por sí mismo para tratar de hallarles solución. Y, en el peor de los casos, estos problemas impuestos le resultarán del todo indiferentes.

Resulta pues muy importante, si se quiere incentivar y ayudar al estudiante a pensar por sí mismo, que el profesor logre plantearle problemas, no desde fuera, sino que surjan de su experiencia, de situaciones que vive o podría vivir. De esta manera, el estudiante puede llegar a sentir y vivir esos problemas como dificultades suyas de las que realmente quiere salir. Sería mejor aún si el profesor en lugar de plantear los problemas por los estudiantes, los guía para que ellos mismos los planteen a partir de situaciones, experiencias o vivencias que los susciten. Aquí se ponen decisivamente en juego la imaginación y creatividad del profesor y la participación activa de sus alumnos.

Es muy probable que si el estudiante contribuye activamente en la formulación del problema que se va a tratar y discutir en el aula de clase, entonces se sienta verdaderamente comprometido a poner en práctica su pensamiento, su inteligencia en la búsqueda de una solución. Por esta razón, el profesor de filosofía que quiera motivar y estimular a sus alumnos a pensar autónomamente, debe estar en capacidad de vincular los problemas filosóficos con las experiencias vitales de ellos. También debe poder orientar a sus alumnos de modo que planteen los problemas de diversas maneras, por medio de diferentes preguntas que permitan ver con claridad cuál es la dificultad que plantean; pues no resultará de mucho provecho comenzar a pensar sobre un problema que aún no se ha comprendido con la suficiente claridad o que no se ha visto todavía como una dificultad que se quiera superar.

Planteado claramente un problema que realmente intrigue a los alumnos y que estén genuinamente interesados en tratar de resolver, pues lo sienten como un problema propio, el profesor puede entonces ayudar a los alumnos a que, en la búsqueda de una solución y en las indagaciones que se realicen para tratar de hallarla o inventarla, ejerciten y desarrollen competencias básicas asociadas con lo que se suele llamar pensamiento crítico. Puesto que los intentos de solución al problema no pueden surgir, por así decirlo, en el vacío, el profesor haría bien en discutir con sus alumnos soluciones que se han intentado dar en la tradición filosófica al 
problema a tratar, a problemas similares o a problemas subsidiarios que se requiera resolver para acercarse a la solución de la dificultad inicial. Si el problema es complejo, suele ser provechoso tratar de dividirlo en preguntas más simples y tratar de indagar acerca de intentos previos de dar respuesta a ellas.

A diferencia de ciertas formas tradicionales de enseñar filosofía, el aprendizaje de problemas, teorías, doctrinas, tesis, argumentos de la tradición filosófica no constituye, en este caso, un fin en sí mismo, sino un medio para aportar elementos que inciten a los alumnos a tratar de formarse una posición propia acerca del problema y de las preguntas con las que está lidiando. La familiarización con ciertos momentos de la historia de la filosofía (y no un recorrido superficial por toda ella) cobraría así un sentido distinto para los estudiantes, pues se trata de aprender a filosofar más que de aprender filosofía (si bien lo segundo se requiere para lo primero). No se trata, pues, de forzarlos a aprender de memoria información que probablemente no les interese, sino de analizarla y examinarla para relacionarla con el problema que se quiere pensar, de manera que contribuya a idear y construir una manera de abordarlo. Aquí el profesor puede dar una buena orientación en la medida que tenga un buen conocimiento y dominio de la historia de la filosofía y de sus principales ámbitos temáticos (tratados) y corrientes. Basado en él puede proponer lecturas de textos filosóficos que sean pertinentes y adecuados que permitan avanzar en el tratamiento del problema y examinar críticamente diversas maneras de abordarlo.

A través del insustituible trabajo con los textos filosóficos (fuentes más que manuales), el profesor debe fomentar las competencias básicas de la lectura crítica: el análisis e interpretación del texto, como su evaluación crítica. En el análisis e interpretación de los textos es importante que el profesor ayude a los alumnos (a través de preguntas adecuadas y sin ahorrarles el trabajo de pensar) a comprender los propósitos principales que se persiguen en el texto y los problemas centrales que se plantean en él; a que analicen la estructura del texto, identificando y distinguiendo las partes principales, subpartes, etc., pero también reconociendo las conexiones entre ellas; a comprender y aclarar los conceptos centrales que se emplean en él y los diferentes sentidos en que se usan; a identificar las tesis centrales que se sostienen y los argumentos o razones que se esgrimen para sustentarlas; a detectar supuestos explícitos y tácitos en los que se apoyan los argumentos; a interpretar y aclarar los pasajes oscuros.
En el examen crítico del texto el profesor debe guiar a los alumnos para que juzgue en qué medida se cumplen los propósitos del mismo; evalúe la fiabilidad de las premisas usadas en los argumentos y la validez de éstos, para lo cual debe distinguir entre diferentes tipos de argumentos (deductivos, inductivos, analogías, argumentos de autoridad, etc.) y diferentes criterios para su evaluación; trate de encontrar objeciones a las tesis centrales del texto o tesis alternativas; identifique posibles incoherencias, ambigüedades $\mathrm{u}$ omisiones en el texto.

El análisis y examen crítico de los textos ha de dar elementos a los alumnos para que intenten elaborar una posición propia sobre el problema en cuestión y para que, sopesando razones a favor y en contra de ella y anticipándose a posibles objeciones, traten de construir argumentos con los que puedan defenderla.

Estas habilidades que son fundamentales para el filosofar (pero que no lo agotan) forman parte de lo que suele llamarse la capacidad de pensar críticamente; esto permite comprender cómo, a través de la enseñanza de la filosofía, o más bien del filosofar, se puede ayudar a los alumnos a fortalecer su pensamiento crítico, y también nos da una idea de lo que ello exige de un profesor de filosofía, a nivel de la educación media.

\section{Referencias}

Adorno, T. (1998). Educación después de Auschwitz. En: Educación para la emancipación. Conferencias y conversaciones con Hellmut Becker (1959-1969). Trad. Jacobo Muñoz. Madrid, España: Ediciones Morata.

Constitución Política de 1991. Bogotá: Congreso de Colombia.

Dewey, J. (1967 [1938]). Experiencia y educación. Buenos Aires: Editorial Losada.

Gadamer, H. (2004). Texto e interpretación (1978). En: Verdad y método II. (Trad. Manuel Olesagasti). Salamanca, España: Ediciones Sígueme.

Grupo de Memoria Histórica (2013). ¡Basta ya! Colombia: Memorias de Guerra y Dignidad. Informe General Grupo de Memoria Histórica. Bogotá: Imprenta Nacional.

Gutmann, A. (2001). La educación democrática: Una teoría política de la educación. Barcelona: Paidós.

Ley 115 de 1994, febrero 8. Por la cual se expide la Ley General de Educación. Bogotá: Congreso de Colombia.

Ministerio de Educación Nacional (MEN). (2010). Orientaciones Pedagógicas para la Filosofía en la Educación Media. Bogotá. 
Ministerio de Educación Nacional (MEN). (2014). Ministerio de educación nacional dirección de calidad para la educación preescolar, básica y media subdirección de referentes y evaluación de la calidad educativa. Bogotá.

Spencer L. y Spencer, S. (1993). Competence at work, models for superior performance. Nueva York: John Wiley \& Sons.

Unesco. (2011). Filosofía: una escuela de la libertad. México: Unesco.

Vargas, G. (2016). El deseo y la formación. Bogotá: Aula de Humanidades. 